http://dx.doi.org/10.21929/abavet2017.73.7

\title{
Pruebas para identificar ovinos resistentes a parásitos gastrointestinales en San Pedro Lagunillas Nayarit
}

\author{
Tests to identify resistant sheep to gastrointestinal parasites in San Pedro Lagunillas \\ Nayarit
}

\author{
Socorro Salgado-Moreno* coco_salgado@hotmail.com, Fernando Carrillo-Díaz** \\ fdoc_8@hotmail.com, Francisco Escalera-Valente franescalera@hotmail.com, Cindy \\ Delgado-Camarena camarena_cindy911@hotmail.com
}

Universidad Autónoma de Nayarit. Unidad Académica de Medicina Veterinaria y Zootecnia. Nayarit, *Autor responsable: Socorro Salgado-Moreno ${ }^{* *}$ Autor de correspondencia: Fernando Carrillo-Díaz Universidad Autónoma de Nayarit. Unidad Académica de Medicina Veterinaria y Zootecnia México.km 3.5 de la carretera Compostela-Chapalilla, Compostela, Nayarit, México. CP. 63700.

\section{RESUMEN}

El presente trabajo se llevó a cabo para determinar a los animales resistentes a nematodos gastroentéricos en ovinos de raza Pelibuey. Para ello se muestreó animales infectados naturalmente y sin desparasitar cada 15 días durante un año. Se utilizó el método FAMACHA como auxiliar para detectar animales anémicos cuando estos están infestados predominantemente por Haemonchus contortus, se tomaron muestras de sangre para realizar microhematocrito, muestras de heces para determinar los parásitos existentes y la carga parasitaria, además se estimó la condición corporal. Las muestras fueron procesadas en los laboratorios de Parasitología y de Análisis Clínicos de la Unidad Académica de Medicina Veterinaria de la UAN. Los valores de hematocrito, carga parasitaria, condición corporal y lectura FAMACHA se utilizaron para determinar la condición de resistencia. Se concluye que el hato en estudio, mantuvo un estado de resistencia a los nematodos gastrointestinales $(70.5 \%)$ durante todo el año. Además, se evidenció que el rebaño mostró más sensibilidad en verano y mayor resistencia en Otoño-Invierno.

Palabras clave: resistencia, FAMACHAC, parásitos gastrointestinales, ovinos.

\begin{abstract}
The present work was carried out to determine the animals resistant to gastroenteric nematodes in Pelibuey sheep. 40 naturally infected ewes without deworming were sampled every 15 days for a year. The FAMACHA method was used to detect anemic animals when they were infested predominantly by Haemonchus contortus, blood samples were taken to make microhematocrit and stool samples to determine the existing parasites and the parasite load. The samples were processed in the Laboratories of Parasitology and Clinical Analysis of the Academic Unit of Veterinary Medicine of the UAN. The values of hematocrit, parasite load, body condition and FAMACHA score were used to determine the resistance condition. It is concluded that the herd under study maintained a state of resistance to gastrointestinal nematodes (70.5\%) throughout the year. In addition, it was evidenced that during the seasons of the year, the herd showed more sensibility in summer and greater resistance in Autumn-Winter.
\end{abstract}

Keywords: resistance, FAMACHA ${ }^{\odot}$, gastrointestinal parasites, sheep.

\section{INTRODUCCIÓN}

La ovinocultura es una actividad pecuaria de gran importancia, debido a la necesidad de satisfacer la demanda creciente de carne ovina para consumo humano. La producción de carne representa el principal fin productivo en varias regiones del mundo; así la carne de ovino constituye una proporción importante en la dieta cárnica 
(García, 2003). Los factores que afectan la rentabilidad de las unidades de producción ovinas son: la raza, reproducción, alimentación y los problemas sanitarios. Dentro de los últimos se encuentran los relacionados con las parasitosis (Quiroz, 2008; Martínez et al., 2011).

En el caso particular de los ovinos, el parasitismo gastrointestinal se considera como una patología que causa mayores pérdidas económicas. Está comprobado que las parasitosis provocan pérdidas en la producción de lana y carne; además de causar muerte en los animales, principalmente los jóvenes. Incluso la producción de leche de oveja, que surge como una alternativa de mercado, no escapa al efecto negativo de los parásitos (Vázquez et al., 2004; Vargas, 2006; López et al., 2010; Martínez et al., 2010 y Quiroz, 2010).

Los nematodos gastrointestinales (NGI), son considerados importantes desde el punto de vista patológico y epidemiológico en diversas zonas geo-ecológicas, principalmente en zonas tropicales, subtropicales y templadas del mundo. La distribución y prevalencia de estos $\mathrm{NGI}$ se debe a la adaptabilidad y resistencia a diversas condiciones climáticas (Vázquez et al., 2004; Cuellar, 2007). Debido a los daños ocasionados por estos organismos, los productores se ven obligados a realizar cuantiosas inversiones para minimizar el efecto negativo al que se ven sometidos sus rebaños (Machen et al., 2002, Schoenian, 2003).

Ante el problema de parasitosis, aparecen métodos de control, como: el uso eficiente de antiparasitarios, manejo de pastoreo, utilización de razas resistentes, vacunas, control biológico con hongos con actividad nematófaga, agujas de cobre, uso de plantas con actividad antihelmíntica (Githiori et al., 2005), interacción entre el parasitismo, el manejo nutricional; y por último el método de desparasitación selectiva llamado FAMACHA ${ }^{\odot}$ (Cuellar, 2007), técnica que puede ser usada como una herramienta auxiliar para identificar la resistencia a infestación parasitaria, principalmente a Haemonchus conturtus (Morales et al., 2002; Morales y Pino, 2009). Asimismo, el uso de FAMACHA demuestra ser una herramienta muy útil para el control del parasitismo gastrointestinal bajo las condiciones del trópico (Morales et al., 2010). También se tienen reportes en cabras que prueban que este método es una estrategia auxiliar para el control de la helmintiasis gastroentéricos en cabras (Ribeiro et al., 2012).

Por otro lado, el método FAMACHA ${ }^{\odot}$ encuentra una correlación entre la coloración de conjuntiva ocular, el valor del paquete celular (VPC) y la presencia del nematodo gastroentérico Haemonchus contortus (Burke, 2005). Ambas, la resistencia a nematodos (definida como la habilidad de un hospedador para iniciar y mantener una respuesta que evite y reduzca el establecimiento de los parásitos, o bien elimine la carga parasitaria) (Albers y Grey, 1987) y la resiliencia (capacidad que tiene un hospedador de mantener casi el mismo nivel de producción ante el desafío parasitario), se ha demostrado que es un factor moderadamente heredable. Una vez que se detectan los animales que no puedan lidiar con la parasitosis, estos pueden ser atendidos y tratados correctamente, lo cual significa dar el fármaco correcto y con las dosis adecuadas (Cuellar, 2007; Martínez et al., 2010). 
El presente trabajo encuentra su justificación si se toman en cuenta las condiciones climáticas y de ubicación del estado de Nayarit, las pérdidas en la producción, el aumento de los costos y el grado de parasitosis que manifiestan los ovinos; por tal motivo se realizó el presente trabajo que tuvo como objetivo el determinar el grado de resistencia del grupo ovino en estudio.

\section{MATERIAL Y MÉTODOS}

El presente trabajo se desarrolló en la "Quinta de Don Ro", ubicada en San Pedro Lagunillas, Nayarit; su clima es cálido subhúmedo y templado lluvioso, con régimen de lluvias de junio hasta diciembre y enero; con una temperatura media anual de $25.6^{\circ} \mathrm{C}$. Tiene una precipitación media anual de $1,210 \mathrm{~mm}^{3}$, de los cuales el $95 \%$ se registra en los meses de julio a septiembre. Los meses más calurosos son de junio a agosto, y los vientos recorren el territorio de oeste a este.

Se tomaron 10 hembras al azar, de un hato de 80 , de raza Pelibuey, con edades de 3 a 5 años, y con un promedio de tres partos por animal. El peso corporal aproximado fue de $55 \mathrm{~kg}$ y una condición corporal (CC) de 2.5 a 3.0 de acuerdo a la escala comparativa morfométrica establecida (en donde el numero 5 corresponde a un animal obeso y el numero 1 a un animal emaciado) (Lucas Tron, 2008).

El tipo de unidad productiva es semi-extensiva, combinando el pastoreo (con duración de 6 a 9 horas diarias) y un complemento (maíz quebrado) en encierro, a razón de 250 gr/animal. Cada 15 días a lo largo de 1 año, a cada animal se le tomó una muestra de heces, directamente del recto, se le extrajo sangre mediante venopunción yugular, utilizando tubos Vacutainer con anticoagulante EDTA y se tomó la lectura FAMACHAC (Bath et al., 2001); y se estimó la condición corporal. A las muestras de heces se les realizó análisis cualitativo con la técnica de Flotación de Willis y conteo fecal de huevos de nematodos por gramo de heces (hgh), mediante la técnica de McMaster modificada. El conteo de hgh permitió establecer el nivel de infestación, siguiendo la clasificación de Morales y Pino (2009). Por otro lado, a las muestras de sangre se le determinó el valor hematocrito (Hto), mediante la técnica de microhematocrito (Morales y Pino 2009).

Para la clasificación de los ovinos en las categorías de resistentes, resilientes y sensibles, se determinó según la metodología de Morales et al. (2010); la cual toma en consideración el nivel de infestación parasitaria, el valor de hematocrito y la lectura FAMACHA ${ }^{\odot}$.

Para determinar si existe diferencia estadística entre los valores de hematocrito y de hgh en los diferentes grupos, se realizó un análisis de varianza; mientras que el contraste de medias se realizó mediante la prueba de Tukey, utilizando el paquete estadístico SPSS, Versión 20.0 (IBM, 2011). 


\section{RESULTADOS}

En el presente trabajo los géneros de parásitos gastroentéricos (NGE) encontrados fueron predominantemente Haemonchus contortus; seguido por Chabertia spp, Cooperia spp, Nematodirus spp, Trichostrongylus spp, Toxocara spp; los géneros de protozoarios Coccidia spp y de los de cestodos Moniezia spp.

La condición resistente fue la que predominó en el hato con el $70.5 \%$ de los animales muestreados, seguida de la condición resiliente con un $20.3 \%$ y por último la condición sensible con el $9.3 \%$ (gráfica 1 ).

Con respecto al hematocrito, se encontró que el porcentaje más alto $(31.7 \%)$ de éste coincide con la condición resistente, el valor intermedio de hematocrito $(30.9 \%)$ con la condición resiliente y el valor Hto más bajo $(21.5 \%)$ con la condición sensible (gráfica 2 ). Las diferencias estadísticas $(P<0.05)$, en cuanto al valor hematocrito, se dieron entre la condición sensible con la resiliente y con la resistente, pero no hubo diferencia entre los dos últimos.

El promedio más alto de huevecillos (1145) se detectó en el grupo resiliente; mientras que el segundo valor (250) se relacionó con el grupo sensible, y por último el promedio más bajo de hgh (103) se determinó en el grupo resistente (grafica 3). Las cargas de hgh mostraron diferencias estadísticas significativas entre el grupo resilientes con el grupo resistente y con el sensible, aunque entre estos dos últimos no se determinó diferencia alguna.

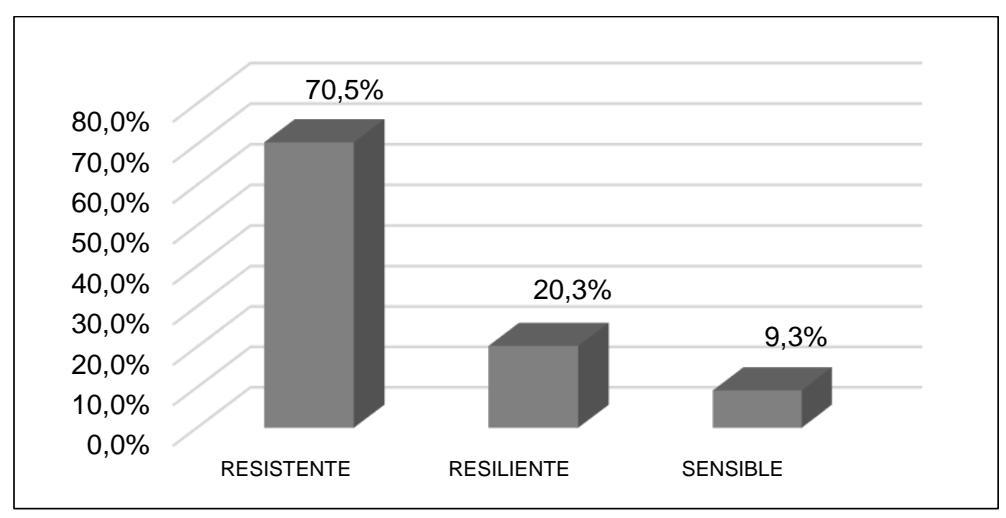

Gráfica I. Descripción del grado de resistencia del hato. 


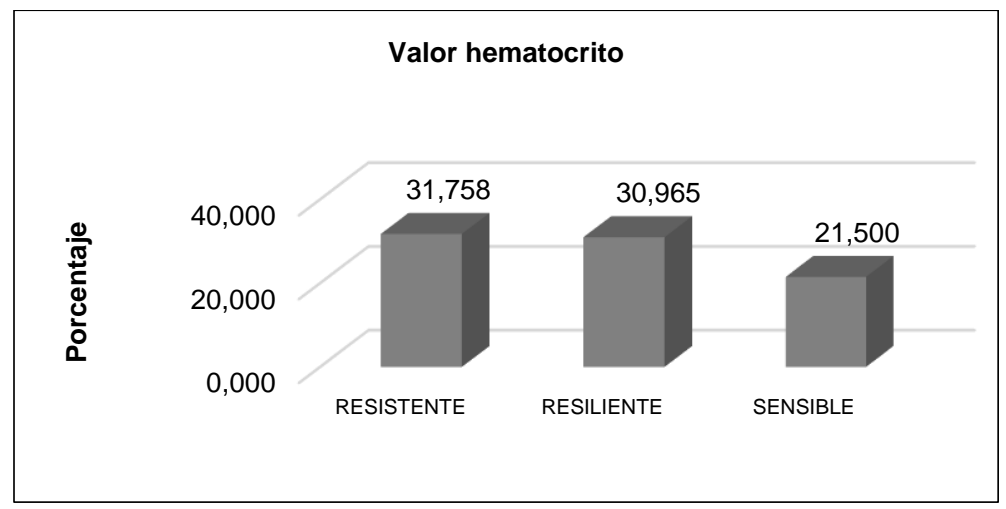

Gráfica II. Valores de hematocrito con base al grado de resistencia.

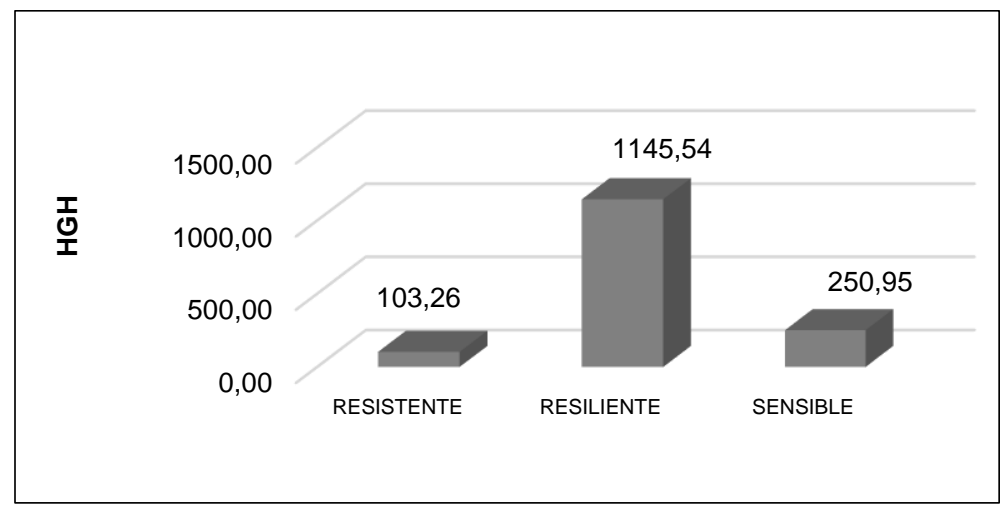

Gráfica III. Promedio de huevecillos por gramo de heces con base al grado de resistencia.

Dentro del grupo resistente el $87.5 \%$ de los animales, se encontró en el nivel óptimo de FAMACHAC. Dentro del grupo resiliente, la mayoría de los animales $(78.3 \%)$ se encontraron en el nivel óptimo; mientras que, dentro del grupo sensible, los porcentajes más altos se concentraron los niveles 3 y 4 de FAMACHA $^{\odot}$, considerados como "Límite" y "Peligroso" respectivamente (Tabla 1).

\begin{tabular}{cccc}
\hline Nivel FAMACHA & Resistente & Resiliente & Sensible \\
\hline 1 (Óptimo) & $87.5 \%$ & $78.3 \%$ & $0.0 \%$ \\
2 (Aceptable) & $11.9 \%$ & $21.7 \%$ & $9.3 \%$ \\
3 (Límite) & $.6 \%$ & $0.0 \%$ & $81.0 \%$ \\
4 (Peligroso) & $0.0 \%$ & $0.0 \%$ & $9.5 \%$ \\
\hline
\end{tabular}

Tabla 1. Clasificación de resistencia de los animales en estudio. 
De acuerdo a la clasificación de resistencia de los animales por época del año, se encontró que los animales fueron más resistentes en otoño e invierno; mientras que el grado de resiliente se detectó en otoño y sensibles en verano (Tabla 2).

\begin{tabular}{ccccccc}
\hline Grado de resistencia & & Primavera & Verano & Otoño & Invierno & Total \\
\hline \multirow{2}{*}{ Resistente } & Recuento & 34 & 34 & 46 & 46 & 160 \\
& $\%$ & $21.3 \%$ & $21.3 \%$ & $28.8 \%$ & $28.8 \%$ & $100.0 \%$ \\
\cline { 2 - 7 } Resiliente & Recuento & 9 & 6 & 19 & 12 & 46 \\
& $\%$ & $19.6 \%$ & $13.0 \%$ & $41.3 \%$ & $26.1 \%$ & $100.0 \%$ \\
\cline { 2 - 7 } Sensible & Recuento & 2 & 15 & 3 & 1 & 21 \\
& $\%$ & $9.5 \%$ & $71.4 \%$ & $14.3 \%$ & $4.8 \%$ & $100.0 \%$ \\
\hline
\end{tabular}

Tabla 2. Clasificación de resistencia de los animales por época del año

\section{DISCUSIÓN}

En Brasil se lleva a cabo un experimento en cabras lecheras, con el objeto de determinar los grados de anemia, utilizando el método $\mathrm{FAMACHA}^{\odot}$ y al igual que en nuestro trabajo este método resulta ser una herramienta auxiliar efectiva para ello; además igual que en el trabajo realizado por nosotros, el parasito predominante fue Haemonchus contortus (Ribeiro et al., 2012).

En Venezuela en el año 2006 Morales y colaboradores llevan a cabo un experimento con bovinos, comparando los niveles de infestación parasitaria, condición corporal y niveles de Hto; encontrando elevadas cantidades de hgh de estrongylos en animales con condición corporal menor o igual a 2.5 y un Hto bajo, y se consideraron susceptibles; mientras que los animales con alta carga de parásitos, CC aceptable (2 a 3) y Hto normal, fueron considerados resilientes. Estos resultados, aunque son en especie bovina, son iguales a los reportados en este trabajo; por tal motivo podemos asumir que el comportamiento de los parásitos en los rumiantes podría calificarse como similar.

Morales et al. en el año 2010 en Venezuela, realizaron una clasificación por el método FAMACHA $^{\odot}$; su relación con el valor del Hto y el recuento de huevos por gramos en ovinos criados en condiciones de pastoreo, validando de forma positiva el uso de la carta de $\mathrm{FAMACHA}^{\odot}$ en el control de parásitos gastrointestinales. Al mismo tiempo reportan que en el rebaño, los ovinos resistentes fueron los dominantes, seguidos de los resilientes y en último término los sensibles.

La metodología de este trabajo es muy similar a la utilizada en esta investigación, con la diferencia que la alimentación era suplementada en corral; asimismo Morales indica que los recuentos de hgh fueron similares entre los animales resilientes y sensibles, sin embargo para los animales resistentes la cuenta fue considerablemente menor; resultados idénticos obtenidos en nuestro trabajo y que además podemos considerar coincidente con lo reportado en la literatura, en que los animales resistentes no son los que tienen una mayor carga parasitaria sin ver afectadas sus parámetros 
productivos, sino los animales que por su condición no permiten el aumento de la carga parasitaria.

En Brasil se evaluó un rebaño de ovinos infectados con NGI, durante un periodo de 912 meses, y se redujo hasta un $86.1 \%$ el número de animales que deberían desparasitar; lo que quería decir que estaban en estado de resistencia o resiliencia muy similar a los datos encontrados en este trabajo (Cuellar, 2007).

También Venezuela Morales (2002), encontró en ovinos de reemplazo, que los mayores valores de hgh correspondieron a los borregos negativos y el valor de Hto, los valores más bajos; para ambos parámetros correspondieron a los animales con niveles altos de infestación, resultados similares a los de este trabajo.

Golberg, (2012) con animales de la raza Merino en el peripato y post destete, realizaron una prueba con el objeto de estimar los parámetros genéticos de resistencia a nematodos; reportando que la mayor eliminación de huevos por las ovejas se observó entre las dos y cuadro semanas post parto, reportando que el Haemonchus spp fue el género predominante en todos los muestreos realizados; los efectos de tipo de parto y edad de la hembra fueron irrelevantes, señalando que es posible seleccionar animales genéticamente resistentes, y que al seleccionar animales jóvenes genéticamente resistentes también se seleccionan animales que eliminaran menor cantidad de huevos. Estos resultados son consistentes con los obtenidos por nosotros, debido a que la mayor cantidad de huevecillos reportados es en el mes de noviembre. Es importante señalar que lo mismo sucede con la edad de la hembra que también fue irrelevante en nuestro trabajo (Golberg, 2012).

En el estado de Guerrero, México se lleva a cabo un trabajo con el fin de conocer los NGI en ovinos en pastoreo, durante los meses de noviembre a enero; concluyendo que los ovinos de este estudio presentaron una alta prevalencia de NGI; de igual forma en nuestro estudio reportamos que la mayor incidencia de NGI la encontramos en el periodo denominado frío (oct-ene), contradiciendo a la creencia tradicional de que durante el periodo de lluvias la carga parasitaria es más alta (Rojas Hernández, 2007).

\section{CONCLUSIÓN}

La metodología empleada para determinar los animales resistentes a NGI en una unidad de producción ovina, resultó ser una herramienta muy útil. El hato en estudio mantuvo un estado resistencia a los nematodos gastroentéricos en un $70.5 \%$ durante todo el año. El rebaño mostró más sensibilidad en verano y mayor resistencia en Otoño-Invierno.

\section{LITERATURA CITADA}

BATH G, Malan FS, Van Wyk JA. The "FAMACHAC". Ovine Anaemia Guide to assist with the control of haemonchosis. In Proceedings of the 7th Annual Congress of Livestock Health and Production Group of South African Veterinary Association, Port Elizabeth, 1996; p. 5-7. 
BURKE J. 2005. Management of barber pole worm in sheep and goats in Southern U.S. Small Farms Research (en línea) fecha de consulta: 10 de marzo 2017 disponible en: https://attra.ncat.org/downloads/goat barber pole.pdf

CUELLAR A. Control no farmacológico de parásitos en ovinos. Desparasitación selectiva por medio del sistema FAMACHA. Tecnologías para ovinocultores. AMCO. 2007; Pp 258-261.

DE LUCAS T. 2009. Fortalecimiento del Sistema de producto Ovinos. Tecnologías para ovinocultores. Serie Producción. Evaluación de la condición corporal en ovejas. (en línea) fecha de consulta: 10 de marzo del 2017 disponible en:

http://www.uno.org.mx/sistema/pdf/produccion/evaluaciondelacondicion.pdf

GARCÍA C. Perspectivas de la ganadería tropical de México ante la globalización. Memoria. XXVII Congreso nacional de Buiatria. Villahermosa, Tabasco. México. 2003; Pp. 45-50.

GITHIORI J. Thamsborg, S and Athanasiadou S. Use of plants in novel approaches to control of gastrointestinal nematodes in small ruminants. Proc. Novel Approaches to Control of Helminths Parasites Livestock. Worm control or worm Management: New paradigms in integrated control. Merida Yucatan, Mexico. 2005.

GOLBERG V, Ciappesoni G, Aguilar I. Modeling the faecal worm egg count curve during the periparturient period in Uruguayan Merino Sheep. Span. J. Agric. Res. 2012; 10(4):986-992. http://dx.doi.org/10.5424/sjar/2012104-3256 .

LÓPEZ M, Vázquez P. Evidencia del alza posparto de huevos de nematodos en ovinos de pelo. Memoria de la Reunión Nacional De Investigación Pecuaria. Villahermosa, Tabasco. México. 2010; Pp. 75-77.

MACHEN R, Craddock F, Graig T, Fuchs,T. A Haemonchus contortus management plan for sheep and goats in Texas. Texas Agriculture Extension Service. The Texas A \& M University System. 2002; Pp 4-98.

MARTíNEZ GS, Aguirre J, Danés A, Ruiz M, Lemus C, Macías H, Moreno S, Ramírez M. Tecnologías para mejorar la producción ovina en México. Revista Fuente. 2010; 2(5). ISSN: 2007 - 0713. http://fuente.uan.edu.mx/publicaciones/02-05/5.pdf

Martínez GS, Macías CH, Moreno FLA, Zepeda GJ, Espinoza MME, Figueroa MR, Ruiz FM. Análisis económico en la producción de ovinos en Nayarit, México. Abanico Veterinario. 2011; 1 (1):37-43. ISSN 2007-4204. http://new.medigraphic.com/cgibin/resumen.cgi?IDARTICULO=45596

MORALES G, Pino L, Rendón Z, Guillen A, Balestrini C, Silva M. Relación entre parámetros hematológicos y el nivel de infestación parasitaria en ovinos de remplazo.

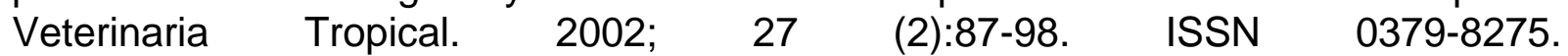
http://sian.inia.gob.ve/revistas ci/VeterinariaTropical/vt2702/arti/morales g.htm

MORALES G, Pino A, Sandoval F, Jiménez D. Niveles de infestación parasitaria, condición corporal y valores de hematocrito en bovinos resistentes, resilientes y 
acumuladores de parásitos en un rebaño criollo Rio Limón. Zootecnia Tropical. 2006; 24(3): 333-346. ISSN

0798-7269. https://dialnet.unirioja.es/servlet/articulo?codigo $=2220485$

MORALES G, Pino L. Nematodos parásitos de los rumiantes domésticos en Venezuela: diagnóstico y control. Editado por Laboratorio de Diagnóstico Veterinario “Aliani”. Impreso en Talleres Graficos Dot Print C>A, Caracas. 2009;143pp

MORALES G, Guillen A, Pino A, Pino L, Barrios F. Clasificación por el método FAMACHA y su relación con el valor de hematocrito y recuento de h.p.g. de ovinos criados en condiciones de pastoreo. Zootecnia tropical. 2010; 28(4):545-555. ISSN 0798-7269. http://www.scielo.org.ve/pdf/zt/v28n4/art11.pdf

QUIROZ R. Parasitología y enfermedades de los animales domésticos. Ed. Limusa, Mexico, D.F. 2008. ISBN : 9789681816742.

ROJAS H, Gutiérrez S, Olivares $\mathrm{P}$, Valencia A. Prevalencia de nemátodos gastrointestinales en ovinos en pastoreo en la parte alta del MPIO. De Cuetzala del Progreso, Guerrero-México Universidad Autónoma de Guerrero - México. REDVET. Revista electrónica de Veterinaria. 2007; 8(9). ISSN 1695-7504.

RIBEIRO V, Ferreira, F., Figueiredo L, Rodrigues A, Beltrao M, Santos A. FAMACHA method as an auxiliary strategy in control of gastrointestinal helmintiasis of dairy goat under semiarid conditions of northeastern. J. Veterinary Parasitology. 2012; 190. 281284. https://doi.org/10.1016/j.vetpar.2012.05.024

SCHOENIAN S. Internal parasite that affect sheep and goats. Maryland Cooperative Extension University of Meryland, USA. 2005; (en línea) Consultado: 10 de marzo 2017 disponible en: http://www.sheepandgoat.com/articles/sheepgoatparasites.pdf

SUÁREZ V. Los parásitos gastrointestinales. Su incidencia en tambo ovino. Salud animal. INTA. Ficha técnica 2007; No. 10. http://inta.gob.ar/sites/default/files/scripttmp-ficha 10-los parasitos gastrointestinales.pdf

VARGAS C. FAMACHA, control de haemonchosis en caprinos. Agronomía mesoamericana. 2006; 17(1):79-88. https://doi.org/10.15517/am.v17i1.5069

VÁZQUEZ V, Flores J, Santiago C, Herrera D, Palacios A, Liabano E, Pelcastre E. Frecuencia de nematodos gastroentéricos en bovinos de tres áreas de clima subtropical húmedo de México. Técnica Pecuaria. 2004; 42(2):327-345. ISSN: 00401889. http://www.redalyc.org/articulo.oa?id=61342209 